\title{
Pilot study: asymptomatic hyperuricemia patients with obesity and nonalcoholic fatty liver disease have increased risk of double contour sign
}

\author{
Hong Ki Min ${ }^{1,2}$, Hyonjoung Cho ${ }^{3}$, and Sung-Hwan Park ${ }^{1}$
}

${ }^{1}$ Division of Rheumatology, Department of Internal Medicine, Seoul St. Mary's Hospital, College of Medicine, The Catholic University of Korea, Seoul; ${ }^{2}$ Division of Rheumatology, Department of Internal Medicine, Konkuk University Medical Center, Seoul; ${ }^{3}$ Division of Rheumatology, Department of Internal Medicine, The Armed Forces Capital Hospital, Armed Forces Medical Command, Seongnam, Korea

Received: December 12, 2018 Revised : March 18, 2019 Accepted: March 19, 2019

\section{Correspondence to}

Sung-Hwan Park, M.D.

Division of Rheumatology,

Department of Internal

Medicine, Seoul St. Mary's Hospital, College of Medicine, The

Catholic University of Korea, 222

Banpo-daero, Seocho-gu, Seoul

06591, Korea

Tel: +82-2-2258-6011

Fax: +82-2-599-3589

E-mail: rapark@catholic.ac.kr
Background/Aims: Double contour sign (DCS) is a representative ultrasonographic finding in gout. DCS is evidence of monosodium urate deposit in gouty arthritis and has been identified in some patients with asymptomatic hyperuricemia. However, the specific characteristics of asymptomatic hyperuricemia in patients with DCS have not yet been revealed.

Methods: We enrolled patients with incidentally found hyperuricemia. Baseline characteristics were compared between asymptomatic hyperuricemia patients with and without DCS. Logistic regression analysis was performed to determine associated factors for DCS in patients with asymptomatic hyperuricemia.

Results: A total of 62 patients with asymptomatic hyperuricemia were enrolled, and 22 of the patients showed DCS. The metatarsophalangeal were the most commonly affected joints, and differences between asymptomatic hyperuricemia patients with and without DCS were seen in aspects of class II obesity and nonalcoholic fatty liver disease (NAFLD). Multivariate logistic regression analysis demonstrated that class II obesity and NAFLD significantly increased the risk of DCS in asymptomatic hyperuricemia patients (odds ratio [OR], 6.58, $p=0.022$; OR, 5.21, $p=0.020$, respectively).

Conclusions: Asymptomatic hyperuricemia patients with class II obesity and NAFLD had increased risk of DCS. Determining the presence of crystal deposition, such as DCS, among patients with asymptomatic hyperuricemia might help determine whether early pharmacologic intervention is needed, especially with severe obesity or NAFLD.

Keywords: Asymptomatic hyperuricemia; Double contour sign; Ultrasonography; Obesity; Non-alcoholic fatty liver disease

\section{INTRODUCTION}

Asymptomatic hyperuricemia is a preliminary condition of gouty arthritis. Although a small percentage of asymptomatic hyperuricemia patients progress to symptomatic gouty arthritis [1], hyperuricemia is the most powerful predictor of gouty arthritis $[2,3]$.
Gout is known to be associated with several chronic diseases [4-6]. Recently, asymptomatic hyperuricemia has been associated with hypertension, chronic kidney disease $(\mathrm{CKD})$, diabetes mellitus, and metabolic syndrome [7-9]. Treatment guidelines for gout suggest using urate lowering therapy (ULT) when gout is confirmed and if accompanied by recurrent flare, tophi, renal stone, 
or urate arthropathy [10]. ULT is a beneficial therapy for gout and prevents recurrent flare ups and resolves tophi. However, indication of ULT in asymptomatic hyperuricemia has not been established.

The Outcome Measures in Rheumatology (OMERACT) Ultrasound Gout Task Force group have proposed four definitions for ultrasonographic elementary lesions in gout, double contour sign (DCS), tophus, hyperechoic aggregates, and erosion [11], although conditions other than DCS had relatively low inter- and intra-reader reliability and the 2015 classification criteria for gout only included DCS on ultrasonographic findings [12,13]. DCS is a definite sign of crystal deposition in hyaline cartilage surface. Therefore, DCS could be assumed as a preliminary lesion of gouty arthritis. Although there are currently no treatment guidelines for asymptomatic hyperuricemia, identifying patients with asymptomatic hyperuricemia who have a preliminary lesion for gouty arthritis could help to select candidates for ULT.

The present study was planned to compare the features of asymptomatic hyperuricemia patients with and without DCS. Additionally, we aimed to identify predictors of DCS in asymptomatic hyperuricemia patients.

\section{METHODS}

\section{Patient and public involvement}

We enrolled patients who came to the Armed Forces Capital Hospital due to incidentally identified hyperuricemia between January and April 2018. Hyperuricemia was defined as serum urate over $7 \mathrm{mg} / \mathrm{dL}$ [14]. Inclusion criteria were as follows: (1) urate level over 7 $\mathrm{mg} / \mathrm{dL}$, (2) age over 18 years, (3) no previous history of arthritis. Patients who take a urate lowering agent or medication which could affect serum urate level such as diuretics, low dose salicylates, angiotensin II receptor blockers, and fenofibrate were excluded [15]. Patients with heavy alcohol drinking were also excluded because alcohol consumption can affect serum urate level [16]. All participants provided written informed consent prior to enrollment. All experiments were conducted in accordance with the Declaration of Helsinki (1964). This study was approved by the Institutional Review Board of the Armed Forces Capital Hospital (AFCH-18-IRB-oo9).

\section{Ultrasonographic findings}

Skeletomuscular ultrasonography was performed by a trained rheumatologist, H.K.M. DCS was defined as an abnormal hyperechoic band over the superficial margin of the hyaline cartilage, independent of the angle of insonation [11]. Ultrasonography was done at six articular joints, the tibiotalar, 1st metatarsophalangeal(MTP), and knee joint medial and lateral femoral condyles, bilaterally. DCS was checked as positive in each joint if at least one side of the joint showed DCS. Ultrasonography was performed in two dimensions by scanning across each joint, moving from medial to lateral and from distal to proximal. The medial and lateral condyles of the femur were assessed in a supine position with full knee flexion. To assess anterior recess of tibiotalar joint and MTP joint, patients were seated on the examination bed with the knee flexed $45^{\circ}$ while placing the foot plantar surface flat [17]. Two readers (H.K.M. and H.C.) decided the presence or absence of DCS independently. When two readers showed a discrepancy between their decisions, they rechecked the ultrasonography. If discordance existed after the re-estimation, an independent assessor (S.H.P.) made the final decision. Ultrasound was done using a multifrequency linear array transducer (8 to 13 $\mathrm{MHz}$ ) of Logiq E (Philips Healthcare, Amsterdam, the Netherlands), on B mode.

\section{Demographic, clinical, and laboratory profiles}

Patient demographic characteristics and laboratory data were collected at the time of first visit. Patient history included information about medication history, alcohol consumption, and smoking status. Comorbidities were investigated by history taking, and nonalcoholic fatty liver disease (NAFLD) was confirmed by liver ultrasonography and exclusion of alcoholic liver disease and viral steatohepatitis. Alcohol consumption was calculated in grams per day by asking the frequency and amount of alcohol consumed at each time. Patients with an alcohol consumption over $40 \mathrm{~g} /$ day were categorized as having alcoholic liver disease [18]. Viral hepatitis markers for hepatitis B and C were checked to rule out steatohepatitis due to viral hepatitis. Dyslipidemia and hypertriglyceridemia were confirmed by checking the lipid profile according to the 2015 Korean Guideline for the management of dyslipidemia [19]. Low density lipoprotein cholesterol over $160 \mathrm{mg} / \mathrm{dL}$ was classified as 
dyslipidemia and triglycerides over $200 \mathrm{mg} / \mathrm{dL}$ as hypertriglyceridemia. Obesity was classified into three stages according to current guidelines from the Korean Society for the Study of Obesity: class I obesity, body mass index (BMI) 25 to 29.9; class II obesity, BMI 30 to 34.9; and class III obesity, BMI $\geq 35$ [20].

\section{Statistical analysis}

Continuous variables were compared by the Mann-Whitney test and displayed as median and interquartile range. Categorical variables were compared between groups using the chi-square test or Fisher's exact test. Inter-reader reliability was estimated by unweighted Cohen's $\kappa$ statistic in terms of categorical assessment (presence or absence of DCS). Cohen's $\kappa$ values of o to 0.20 were considered as poor, 0.21 to 0.40 fair, 0.41 to 0.60 moderate, 0.61 to 0.80 good and $>0.80$ excellent. Univariate and multivariate logistic regression analysis were performed, and factors that yielded a $p<0.05$ by univariate analysis were considered for the multivariate analysis. Values of $p<0.05$ were considered statistically significant. All tests were performed using R for Windows 3.3.2 (The R Foundation for Statistical Computing, Vienna, Austria).

\section{RESULTS}

\section{Patient baseline characteristics and comparison between asymptomatic hyperuricemia patients with and without DCS}

A total of 62 patients with asymptomatic hyperuricemia were enrolled. All enrolled patients were male, and the median age was 38 years. Total 372 joints (six sites for each patient) was examined, and DCS was observed in 24 sites out of 22 of the enrolled patients, and MTP was the most common site (Table 1). Representative ultrasonographic images of DCS in each joint are shown in Fig. 1. Cohen's $\kappa$ value for existence of DCS was $0.78(p=$ o.016). Class II obesity and NAFLD were more frequent in asymptomatic hyperuricemia patients with DCS than in those without DCS (38.1\% vs. 7.9\%, $p=0.012 ; 50.0 \%$ vs. $12.81 \%, p=0.004$, respectively) (Table 1 ).

\section{Factors associated with DCS in patients with asymp- tomatic hyperuricemia}

Univariate and multivariate logistic regression analysis were conducted to identify factors associated with DCS. On univariate analysis, class II obesity (odds ratio [OR], 7.18; $p=0.009$ ), NAFLD (OR, 6.80; $p=0.003$ ), and smoking (OR, 3.52; $p=0.033$ ) significantly increased the risk of DCS in patients with asymptomatic hyperuricemia. In multivariate analysis, class II obesity and NAFLD significantly increased the risk of DCS (Table 2).

\section{DISCUSSION}

Gout and asymptomatic hyperuricemia have been a recent focus because chronic diseases such as hypertension, CKD, and metabolic syndrome have been associated with gout and with asymptomatic hyperuricemia [4-9,21]. Previous discussion has raised the question that ULT might have beneficial effects not only on preventing gout flare, but also on related chronic diseases. One prospective study showed that ULT attenuated renal damage in asymptomatic hyperuricemia patients with CKD [22]. However, evidence that supports the beneficial effects of ULT in asymptomatic hyperuricemia is insufficient, and internationally acceptable treatment guidelines have not yet been established. Dalbeth and Stamp [23] the stage of gout and asymptomatic hyperuricemia according to symptoms and ultrasonographic evidence of monosodium urate (MSU) deposit and proposed that early intervention for patients with asymptomatic hyperuricemia with MSU deposits could potentially have a preventive role. It is important to identify and select candidates among patients with asymptomatic hyperuricemia for ULT to prevent symptomatic gouty arthritis and related diseases. The present study showed that class II obesity and NAFLD were significantly associated with the presence of DCS in patients with asymptomatic hyperuricemia, and this finding might aid in selecting candidates with ULT.

The OMERACT Ultrasound Gout Task Force group has suggested four elementary lesions in gout: tophi, hyperechoic aggregate, erosion, and DCS [11]. However, recent classification criteria for gout have only included DCS as an ultrasonographic finding [12]. Erosion proven by conventional radiography is also included in classification criteria, but it is a relatively common feature in inflammatory arthritis. Tophi are commonly seen in the synovium or tendon in cases of gout, but a recently pub- 
Table 1. Comparison between asymptomatic hyperuricemia patients with or without double contour sign

\begin{tabular}{|c|c|c|c|c|}
\hline Variable & $\begin{array}{l}\text { Total asymptomatic } \\
\text { hyperuricemia } \\
\text { patients } \\
(\mathrm{n}=62)\end{array}$ & $\begin{array}{l}\text { Asymptomatic hyperuricemia } \\
\text { patients without double } \\
\text { contour sign } \\
(\mathrm{n}=4 \mathrm{O})\end{array}$ & $\begin{array}{l}\text { Asymptomatic } \\
\text { hyperuricemia with } \\
\text { double contour sign } \\
\qquad(\mathrm{n}=22)\end{array}$ & $p$ value \\
\hline Age, yr & $38.5(24.0-49.0)$ & $37.5(24.0-46.5)$ & $39.0(23.0-49.0)$ & 0.912 \\
\hline $\mathrm{BMI}, \mathrm{kg} / \mathrm{m}^{2}$ & $26.7(25.2-28.7)$ & $26.2(24.0-28.6)$ & $27.7(26.0-30.1)$ & 0.050 \\
\hline Obesity $(\mathrm{BMI} \geq 25)$ & $45(76.3)$ & $26(68.4)$ & $19(90.5)$ & 0.113 \\
\hline Class II obesity $(\mathrm{BMI} \geq 30)$ & $11(18.6)$ & $3(7.9)$ & $8(38.1)$ & 0.012 \\
\hline Smoking & $18(30.0)$ & $8(20.5)$ & $10(47.6)$ & 0.059 \\
\hline Family history of gout & $8(13 \cdot 3)$ & $5(12.8)$ & $3(14 \cdot 3)$ & 1.000 \\
\hline HBP & $17(27.9)$ & $10(25.6)$ & $7(31.8)$ & 0.826 \\
\hline $\mathrm{DM}$ & $4(6.6)$ & $2(5 \cdot 1)$ & $2(9.1)$ & 0.951 \\
\hline Dyslipidemia & $17(27.9)$ & $10(25.6)$ & $7(31.8)$ & 0.826 \\
\hline $\mathrm{CKD}$ & $2(3 \cdot 3)$ & $2(5.1)$ & 0 & 0.740 \\
\hline NAFLD & $16(26.2)$ & $5(12.8)$ & $11(50.0)$ & 0.004 \\
\hline Alcohol consumption, g/day & $2.8(0.0-10.8)$ & $2.9(0.0-10.8)$ & $1.4(0.0-10.8)$ & 0.721 \\
\hline $\mathrm{ESR}, \mathrm{mm} / \mathrm{hr}$ & $6.0(3.0-10.0)$ & $5.0(2.5-8.0)$ & $7.0(3.0-11.0)$ & 0.253 \\
\hline $\mathrm{CRP}, \mathrm{mg} / \mathrm{dL}$ & $0.1(0.1-0.2)$ & $0.1(0.1-0.2)$ & $0.2(0.1-0.3)$ & 0.308 \\
\hline Uric acid, mg/dL & $9.6(8.5-10.5)$ & $9.8(8.6-10.6)$ & $9.1(8.3-10.3)$ & 0.317 \\
\hline Uric acid $\geq 9 \mathrm{mg} / \mathrm{dL}$ & $40(64 \cdot 5)$ & $27(67.5)$ & $13(59.1)$ & 0.700 \\
\hline Glucose, mg/dL & $90.5(86.0-96.0)$ & $90.0(87.0-95.0)$ & $91.0(82.5-96.0)$ & 0.827 \\
\hline BUN, mg/dL & $14.3(12.0-16.7)$ & $14.2(12.8-16.7)$ & $14.8(11.0-17.9)$ & 0.877 \\
\hline Creatinine, mg/dL & $0.9(0.8-1.0)$ & $0.9(0.8-1.0)$ & $0.9(0.8-1.1)$ & 0.702 \\
\hline AST, IU/L & $28.5(24.0-35.0)$ & $26.0(21.0-32.0)$ & $33.0(29.0-37.0)$ & 0.014 \\
\hline ALT, IU/L & $32.0(23.0-53.0)$ & $29.0(21.0-38.0)$ & $50.0(27.0-61.0)$ & 0.008 \\
\hline LDL-C, mg/dL & $120.0(105.0-131.0)$ & $120.0(107.5-137.5)$ & $110.5(92.0-121.0)$ & 0.062 \\
\hline Triglyceride, mg/dL & $163.0(115.0-273.0)$ & $161.0(102.0-273.0)$ & $210.0(136.0-262.0)$ & 0.408 \\
\hline Hypertriglyceridemia & $25(58.1)$ & $16(57.1)$ & $9(60.0)$ & 1.000 \\
\hline Double contour sign & $24 / 372(6.45)$ & & & \\
\hline MTP joint & 13 & & & \\
\hline Knee & 8 & & & \\
\hline Ankle & 3 & & & \\
\hline
\end{tabular}

Values are presented as median (interquartile range) or number (\%).

BMI, body mass index; HBP, hypertension; DM, diabetes mellitus; CKD, chronic kidney disease; NAFLD, nonalcoholic fatty liver disease; ESR, erythrocyte sedimentation rate; CRP, C-reactive protein; BUN, blood urea nitrogen; AST, aspartate aminotransferase; ALT, alanine aminotransferase; LDL-C, low density lipoprotein cholesterol; MTP, metatarsophalangeal.

lished systemic review revealed that these findings had relatively low sensitivity for gouty arthritis [13]. Several studies evaluated the presence of DCS in patients with asymptomatic hyperuricemia $[14,24]$. One study revealed that DCS was found in $25 \%$ and $17 \%$ of 1 st MTP and femoral hyaline cartilage, respectively [14]. The present study showed similar results that $35.5 \%$ of patients with asymptomatic hyperuricemia had DCS, and that the MTP was the most frequently affected joint.

Uric acid level is the most powerful predictor of development from asymptomatic hyperuricemia to gouty arthritis, but other factors, such as excessive weight gain 

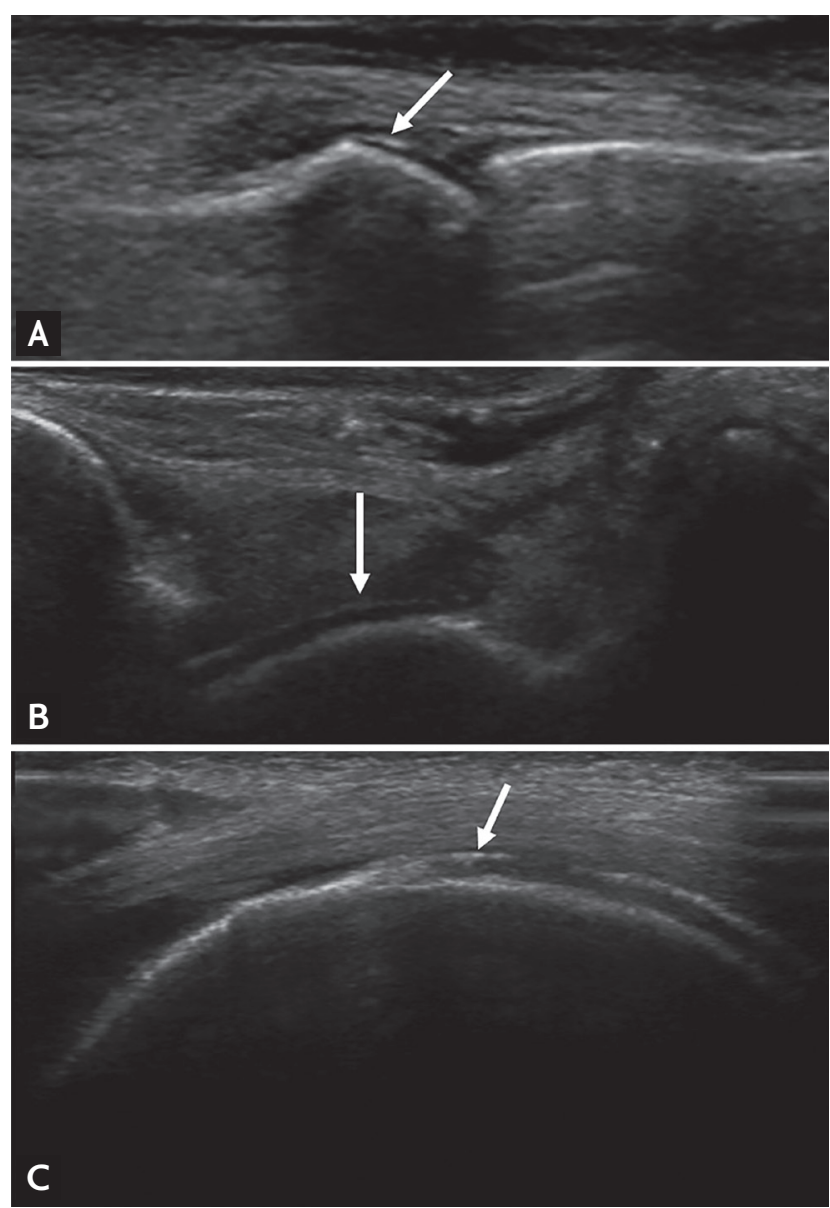

Figure 1. Representative ultrasonographic images of double contour sign (white arrows) in (A) 1st metatarsophalangeal, (B) tibiotalar, and (C) knee joint.

also affect the occurrence of symptomatic gouty arthritis [2]. The present study showed that class II obesity was significantly associated with presence of DCS. Adipose tissue secretes various adipokines, and adiponectin was negatively associated with urate level [25]. However, the effect of adipokines on crystal deposits has not yet been elucidated. Further basic research could reveal whether adipokine and obesity have pathologic role on crystal deposit or not.

Most patients with NAFLD are obese, but the present study showed a modest correlation between class II obesity and NAFLD (five NAFLD among 11 class II obesity patients, and five class II obesity cases among 16 NAFLD patients). To clarify and confirm the independent relationships between NAFLD, obesity, and crystal deposition, further studies on the mechanism between obesity, NAFLD, and crystal deposition should be investigated.
And it would be interesting research if follow-up data reveals the therapeutic effect of treating obesity and NAFLD on DCS. Interestingly, the most powerful predictor of gout flare, serum uric acid level, was not associated with DCS. However, in the present study, uric acid level was measured only once, and this is not representative of the average urate level. Duration of hyperuricemia state and mean level of serum uric acid may be more important on formation of crystal deposit, and it could be assessed by measuring urate levels over multiple time points.

There were several limitations to this study. First, the present study was performed by cross-sectional analysis, therefore, the causality between obesity and NAFLD on DCS could not be proven. Second, the enrolled study population was relatively small. Third, the enrolled patients were all male and relatively young; therefore, the impact of obesity and NAFLD on female or elderly patients with asymptomatic hyperuricemia could not be evaluated. Forth, the joint fluid analysis was not performed in patients with DCS. Therefore, discriminating MSU from other crystal such as calcium pyrophosphate crystal was impossible in present study. Finally, the present study did not include other ultrasonographic findings for gout such as tophi and hyperechoic aggregates. Although DCS is the most representative finding in gout, there are several limitations with respect to confirming DCS. The detection rate for DCS depends on the sonographer's proficiency, and inter-observer reliability is relatively variable. Despite the limitations, this was the first study which demonstrated risk factors for ultrasonographic proven crystal deposits in asymptomatic hyperuricemia patients.

In conclusion, several differences were observed between asymptomatic hyperuricemia patients with and without DCS. Accompanying class II obesity and NAFLD were significantly associated with DCS in asymptomatic hyperuricemia patients. This association could be used to identify asymptomatic hyperuricemia patients who have preliminary lesions and that could benefit from ULT. Patients with asymptomatic hyperuricemia who have severe obesity and NAFLD could require ULT to prevent symptomatic gouty arthritis and to resolve crystal deposits. 
Table 2. Logistic regression analysis for finding related variables of double contour sign

\begin{tabular}{|c|c|c|c|c|c|c|}
\hline \multirow{2}{*}{ Variable } & \multicolumn{3}{|c|}{ Univariate } & \multicolumn{3}{|c|}{ Multivariate } \\
\hline & OR & $95 \% \mathrm{CI}$ & $p$ value & OR & $95 \% \mathrm{CI}$ & $p$ value \\
\hline Age, yr & 1.01 & $0.96-1.05$ & 0.808 & & & \\
\hline $\mathrm{BMI}, \mathrm{kg} / \mathrm{m}^{2}$ & 1.15 & $0.97-1.36$ & 0.117 & & & \\
\hline Obesity & 4.38 & $0.88-21.93$ & 0.072 & & & \\
\hline Class II obesity & 7.18 & $1.65-31.28$ & 0.009 & 6.58 & $1.31-33.02$ & 0.022 \\
\hline Smoking & 3.52 & $1.11-11.20$ & 0.033 & 3.04 & $0.77-11.90$ & 0.111 \\
\hline Family history of gout & 1.13 & $0.24-5.29$ & 0.874 & & & \\
\hline HBP & 1.35 & $0.43-4.27$ & 0.606 & & & \\
\hline $\mathrm{DM}$ & 1.85 & $0.24-14.14$ & 0.553 & & & \\
\hline Dyslipidemia & 1.35 & $0.43-4.27$ & 0.606 & & & \\
\hline NAFLD & 6.80 & $1.93-23.90$ & 0.003 & 5.21 & $1.30-21.00$ & 0.020 \\
\hline $\mathrm{ESR}, \mathrm{mm} / \mathrm{hr}$ & 1.04 & $0.98-1.10$ & 0.232 & & & \\
\hline CRP, mg/dL & 2.59 & $0.76-8.83$ & 0.129 & & & \\
\hline Uric acid, mg/dL & 0.93 & $0.68-1.29$ & 0.668 & & & \\
\hline Uric acid $\geq 9 \mathrm{mg} / \mathrm{dL}$ & 0.70 & $0.24-2.04$ & 0.509 & & & \\
\hline AST, IU/L & 1.02 & $0.99-1.05$ & 0.238 & & & \\
\hline $\mathrm{ALT}, \mathrm{IU} / \mathrm{L}$ & 1.01 & $0.99-1.02$ & 0.465 & & & \\
\hline Hypertriglyceridemia & 1.12 & $0.31-4.03$ & 0.856 & & & \\
\hline
\end{tabular}

OR, odd ratio; CI, confidence interval; BMI, body mass index; HBP, hypertension; DM, diabetes mellitus; NAFLD, nonalcoholic fatty liver disease; ESR, erythrocyte sedimentation rate; CRP, C-reactive protein; AST, aspartate aminotransferase; ALT, alanine aminotransferase.

\section{KEY MESSAGE}

1. Asymptomatic hyperuricemia patients with class II obesity and nonalcoholic fatty liver disease have increased risk of double contour sign (DCS).

2. Discriminating asymptomatic hyperuricemia patients who have definite crystal deposit such as DCS may help to select candidates for urate lowering therapy.

\section{Conflict of interest}

No potential conflict of interest relevant to this article was reported.

\section{REFERENCES}

1. Campion EW, Glynn RJ, DeLabry LO. Asymptomatic hy- peruricemia. Risks and consequences in the Normative Aging Study. Am J Med 1987;82:421-426.

2. Lin KC, Lin HY, Chou P. The interaction between uric acid level and other risk factors on the development of gout among asymptomatic hyperuricemic men in a prospective study. J Rheumatol 2000;27:1501-1505.

3. Dalbeth N, Phipps-Green A, Frampton C, Neogi T, Taylor WJ, Merriman TR. Relationship between serum urate concentration and clinically evident incident gout: an individual participant data analysis. Ann Rheum Dis 2018;77:1048-1052.

4. Roughley MJ, Belcher J, Mallen CD, Roddy E. Gout and risk of chronic kidney disease and nephrolithiasis: meta-analysis of observational studies. Arthritis Res Ther 2015;17:90.

5. Gonzalez-Senac NM, Bailen R, Torres RJ, de Miguel E, Puig JG. Metabolic syndrome in primary gout. Nucleosides Nucleotides Nucleic Acids 2014;33:185-191.

6. Abeles AM. Hyperuricemia, gout, and cardiovascular disease: an update. Curr Rheumatol Rep 2015;17:13. 
7. Li L, Yang C, Zhao Y, Zeng X, Liu F, Fu P. Is hyperuricemia an independent risk factor for new-onset chronic kidney disease? A systematic review and meta-analysis based on observational cohort studies. BMC Nephrol 2014;15:122.

8. Wang J, Qin T, Chen J, et al. Hyperuricemia and risk of incident hypertension: a systematic review and meta-analysis of observational studies. PLoS One 2014;9:e114259.

9. Li C, Hsieh MC, Chang SJ. Metabolic syndrome, diabetes, and hyperuricemia. Curr Opin Rheumatol 2013;25:210216.

10. Richette P, Doherty M, Pascual E, et al. 2016 Updated EULAR evidence-based recommendations for the management of gout. Ann Rheum Dis 2017;76:29-42.

11. Gutierrez M, Schmidt WA, Thiele RG, et al. International consensus for ultrasound lesions in gout: results of Delphi process and web-reliability exercise. Rheumatology (Oxford) 2015:54:1797-1805.

12. Neogi T, Jansen TL, Dalbeth N, et al. 2015 Gout classification criteria: an American College of Rheumatology/ European League Against Rheumatism collaborative initiative. Ann Rheum Dis 2015;74:1789-1798.

13. Ogdie A, Taylor WJ, Weatherall M, et al. Imaging modalities for the classification of gout: systematic literature review and meta-analysis. Ann Rheum Dis 2015;74:18681874 .

14. Pineda C, Amezcua-Guerra LM, Solano C, et al. Joint and tendon subclinical involvement suggestive of gouty arthritis in asymptomatic hyperuricemia: an ultrasound controlled study. Arthritis Res Ther 2011;13:R4.

15. Ben Salem C, Slim R, Fathallah N, Hmouda H. Drug-induced hyperuricaemia and gout. Rheumatology (Oxford) 2017;56:679-688.

16. Towiwat P, Li ZG. The association of vitamin C, alcohol, coffee, tea, milk and yogurt with uric acid and gout. Int J Rheum Dis 2015;18:495-501.

17. Martinoli C. Musculoskeletal ultrasound: technical guidelines. Insights Imaging 2010;1:99-141.

18. Stickel F, Datz C, Hampe J, Bataller R. Pathophysiology and management of alcoholic liver disease: update 2016. Gut Liver 2017;11:173-188.

19. Committee for the Korean Guidelines for the Management of Dyslipidemia. 2015 Korean guidelines for the management of dyslipidemia: executive summary (English translation). Korean Circ J 2016;46:275-306.

20. Kim MK, Lee WY, Kang JH, et al. 2014 Clinical practice guidelines for overweight and obesity in Korea. Endocrinol Metab (Seoul) 2014;29:405-409.

21. Thottam GE, Krasnokutsky S, Pillinger MH. Gout and metabolic syndrome: a tangled web. Curr Rheumatol Rep 2017;19:60.

22. Sircar D, Chatterjee S, Waikhom R, et al. Efficacy of febuxostat for slowing the GFR decline in patients with CKD and asymptomatic hyperuricemia: a 6-month, double-blind, randomized, placebo-controlled trial. Am J Kidney Dis 2015;66:945-950.

23. Dalbeth N, Stamp L. Hyperuricaemia and gout: time for a new staging system? Ann Rheum Dis 2014;73:1598-1600.

24. Stewart S, Dalbeth N, Vandal AC, Allen B, Miranda R, Rome K. Ultrasound features of the first metatarsophalangeal joint in gout and asymptomatic hyperuricemia: comparison with normouricemic individuals. Arthritis Care Res (Hoboken) 2017;69:875-883.

25. Bo S, Gambino R, Durazzo M, et al. Associations between serum uric acid and adipokines, markers of inflammation, and endothelial dysfunction. J Endocrinol Invest 2008;31:499-504. 